\title{
The Macrocycle Plane Orientation of Phthalocyanine and Porphyrin Derivatives on an Air-water Interface
}

\author{
Hai-Xia Xiao ${ }^{1}$, Xu-Xin Cheng ${ }^{1, a}$, Shi-Quan Xi ${ }^{2}$ and Hai-Ning Cui 1, 2, b* \\ ${ }^{1}$ College of Electronic Information and Mechatronic engineering, Zhaoqing University (526061) , \\ Guangdong, PR China \\ ${ }^{2}$ Changchun Institute of Applied Chemistry, Chinese Academy of Sciences, China \\ acxx101@126.com, b751211507@qq.com
}

\begin{abstract}
Keywords: Copper Phthalocyanine film, Porphyrin film, Monomolecular Layer, structural characterization.

Abstract. Systematic research on monomolecular layer of phthalocyanine (Pc) and porphyrin (Por) derivatives bas been finished. In this paper an attention is focused upon the orientation and characterization of molecular macrocycle planes of some Pc and Por derivatives in monomolecular layer by means of the the surface pressure-area $(\pi-\mathrm{A})$ isotherm method .
\end{abstract}

\section{Introduction}

Phthalocyanine (Pc) and porphyrin (Por) derivatives, as highly conjugated and symmetric disk-shaped molecules, exhibit a wide variety of physical, chemical and electrical properties. The monolayer and nanometer assembly films of these macrocycle derivatives by means of Langmuir-Blogdett (LB) technique allow functional molecules to be arranged in a highly ordered structure and offer the possibility of application in many aspects[1-3]. Since structural studies of Pc and Por films have fallen far behind to the studies of their physical properties, of particular interests are is focused upon determination of the orientation of molecular macrocycle plane in the film based on the traditional methods and improve technique .

\section{Experiments and Preparation of the Film}

For improving the film forming properties the copper phthalocyanine $(\mathrm{CuPc})$ and Por derivatives synthesized were as following. (1) Symmetrically substituted macrocycles. (2) Asymmetrically substituted macrocycles, as shown in Table 1. CuPc were prepared according to the procedures reported by Snow et al [4-5]. Details of the preparation and characterization were described elsewhere[5-6]. Porphyrins were prepared according to the method reported in our previous paper [7]. KSV-5000 Langmuir trough (Finland) with a Wilhelmy balance and domestic LB trough were employed for the surface pressure-area (Pi-A) isotherm measurements and the LB films fabrication.

\section{Studies of the Orientation of Macrocycle Planes}

The molecular orientation of various Pc and Por macrocycle plane in ordered films has systemically been studied by the information of area per molecule from $\mathrm{Pi}-\mathrm{A}$ isotherm. Among these $\mathrm{Pi}-\mathrm{A}$ isotherms representative isotherm of $\mathrm{CuPc}[\mathrm{dtpp}]_{4}$ and TPIATPP are shown in Fig. 1. The collapse pressure of molecules and the area per molecule obtained by extrapolating the Pi-A curve ( or the limiting area at zero pressure) and other properties of $\mathrm{CuPc}$ and Por derivatives listed in Table 2. It is seen that the area occupied by per molecule in the films of asymmetrically substituted $\mathrm{CuPc}$ is larger than that of symmetrically substituted $\mathrm{CuPc}$, where the former differs from the latter in possessing long and asymmetrically hydrophilic substituents. These polar groups would render the greater adhesion of macrocycle plane face to the water and lesser cohesion between ring planes. As the minimum plane area occupied by macrocycle of $\mathrm{CuPc}$ molecule would be $1.6 \mathrm{~nm} 2$ and the surface area of the ring edge is $0.4-0.8 \mathrm{~nm}^{2}$ [8]. According to the area per molecule found in our study, the monomolecular area for $\mathrm{CuPc}$ in film are less than that of real CuPc macrocycle plane area, and the measured $\mathrm{CuPc}$ area of $0.45-1.2 \mathrm{~nm}^{2}$ is incompatible with an arrangement of the macrocycle plane 
parallel to the water surface for which values near $1.6 \mathrm{~nm}^{2}$ are measured. In the same reason the rings are not perpendicular to the surface either. These indicate a tilted of the planes of macrocycle with respect to one another as sketched in Fig. 2. For porphyrins, the area occupied by per molecule in the films of natural source porphyrin-asymmetrically PP and near symmetrically substituted Porphyrin C is similar. In addition for case of four symmetrically substituted groups, the area occupied by per molecule in the films of TPIATPP is larger than that of CdOClTPP with substituted central metal ion-Cd, where the former differs from the latter in possessing long substituent chains.

\section{Table 1. Schematic structure of the copper phthalocyanine and Porphyrin derivatives}

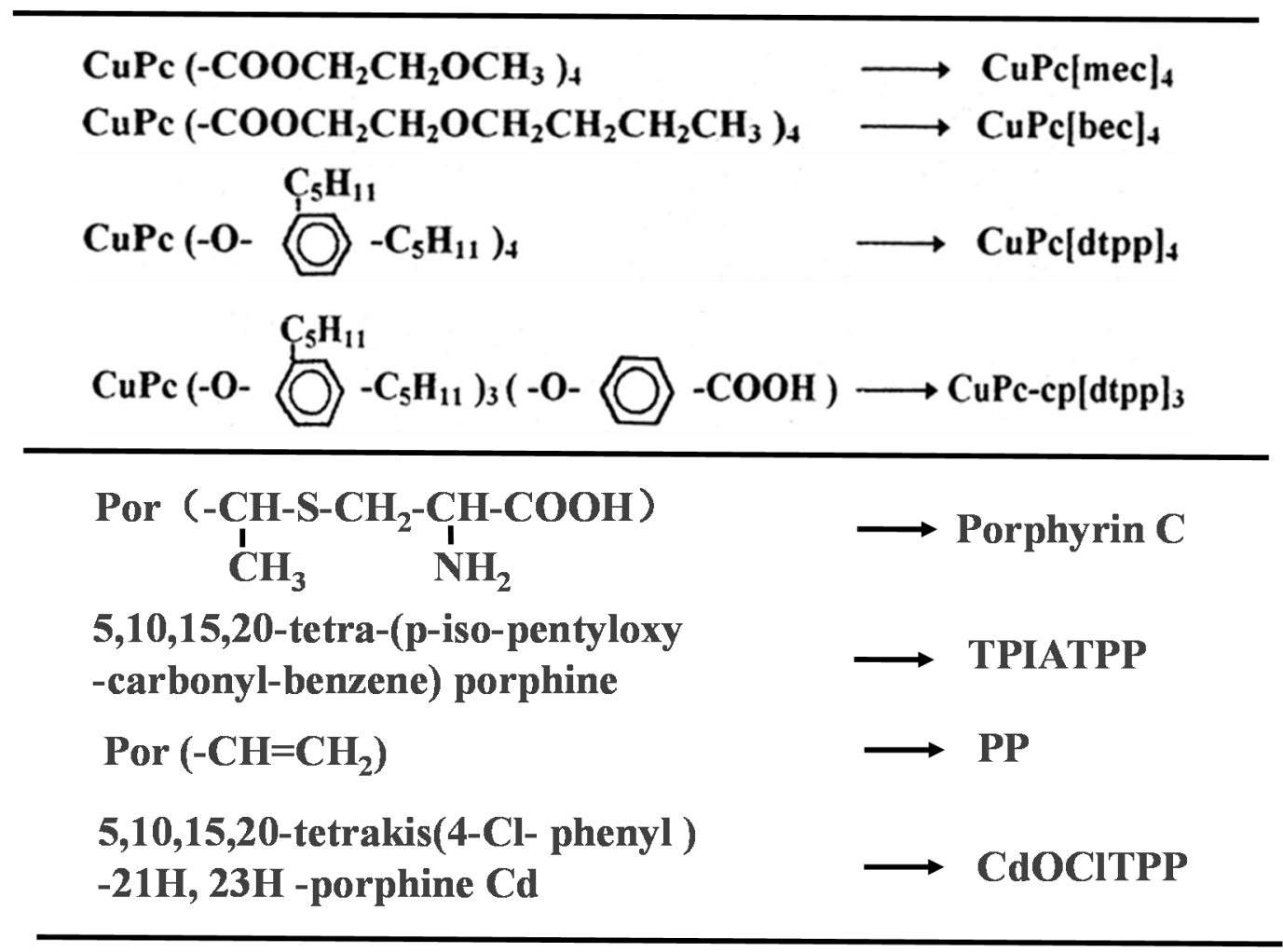

Some explanations of above is as follows: 1. While Pc and Por molecules are laid on the water surface at zero pressure, the macrocycle prefer to 'lie flat'. Because in initial spreading process on the air-water 'lie flat' tendency of the ring plane is produced by the intermolecular and varied external force function, especially the hydrophilic carboxyl group have tend to water surface. This can be deduce through the analysis of construction of the molecular model and mechanics. It is that the equilibrium position of the macrocycle plane model should indeed lie flat, and later as surface pressure increases, the hydrophilic carboxyl group stays in the water while the rest of the molecule is pushed up by external force. Final the plane becomes 'tilted'. 2 . The maintenance of such a vertical orientation of the ring planes in the presence of the water surface implies that there are strong attractive interactions between the rings that tend to make them assume a card-pack orientation. Hence, when the surface pressure increases further, the ring plane model only becomes tightly under the mechanics rules. 


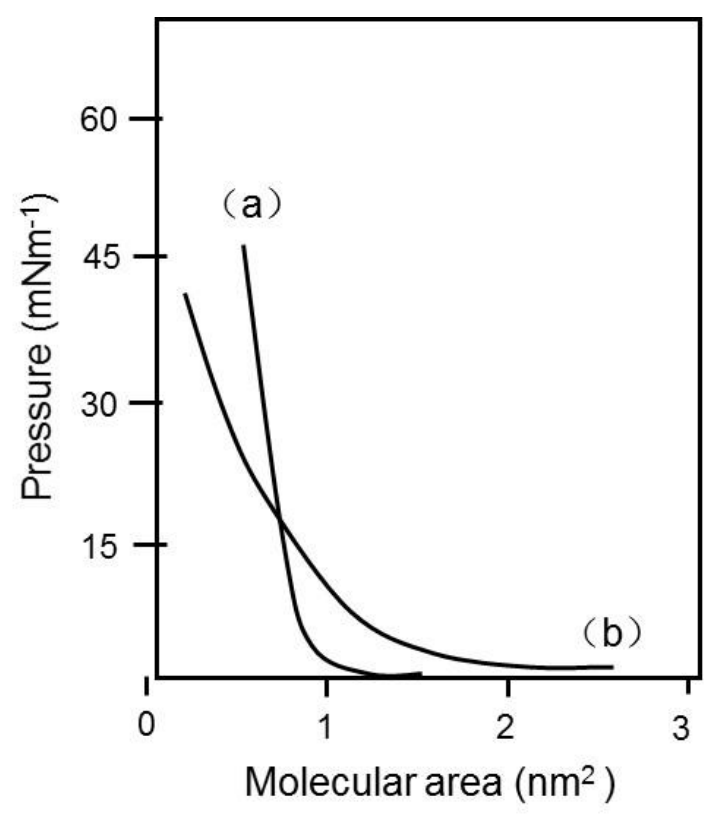

Fig. 1 A normal surface pressure-area (p-A) isotherm of $\mathrm{Pc}$ and Por ; (a) CuPc[dtpp $] 4$; (b) TPIATPP.

Table 2. Parameters of the $\pi-\mathrm{A}$ isotherm about $\mathrm{CuPc}$ and Porphyrin layer on air-water interface

\begin{tabular}{|c|c|c|c|c|c|}
\hline \multirow{4}{*}{$\begin{array}{l}\text { Samples } \\
\text { Transfer }\end{array}$} & \multirow{4}{*}{$\begin{array}{l}\text { Collapse } \\
\text { Pressure } \\
\text { mNm}^{-1}\end{array}$} & \multirow{4}{*}{$\begin{array}{l}\text { Select } \\
\text { Transfer } \\
\mathrm{mNm}^{-1}\end{array}$} & \multicolumn{3}{|c|}{ The occupied area per molecule $\left(\mathrm{nm}^{2}\right)$} \\
\hline & & & \multirow{3}{*}{$\begin{array}{l}\text { Under Zero } \\
\text { Pressure }\end{array}$} & \multirow{3}{*}{$\begin{array}{r}\text { Under } \\
\text { Pressure }\end{array}$} & \multirow{3}{*}{$\begin{array}{r}\text { Collapse } \\
\text { P }\end{array}$} \\
\hline & & & & & \\
\hline & & & & & \\
\hline $\mathrm{CuPc}[\mathrm{mec}]_{4}$ & 36 & 25 & 1.01 & 0.45 & 0.70 \\
\hline $\mathrm{CuPc}[\mathrm{bec}]_{4}$ & 36 & 25 & 1.02 & 0.51 & 0.72 \\
\hline $\mathrm{CuPc}[\mathrm{dtpp}]_{4}$ & 43 & 30 & 1.01 & 0.48 & 0.64 \\
\hline CuPc-cp $[\mathrm{dtpp}]_{3}$ & 44 & 30 & 1.20 & 0.45 & 0.75 \\
\hline Porphyrin C & 43 & 30 & 1.15 & 0.41 & 0.51 \\
\hline TPIATPP & 42 & 30 & 1.60 & 0.22 & 0.78 \\
\hline PP & 44 & 25 & 1.20 & 0.40 & 0.76 \\
\hline CdOClTPP & 51 & 25 & 0.64 & 0.20 & 0.45 \\
\hline
\end{tabular}




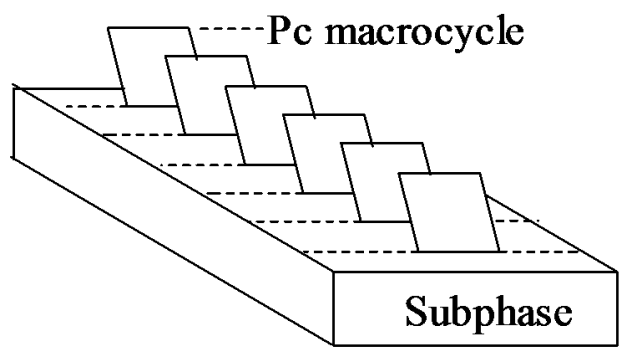

Fig. 2 Sketch of the arrangement of CuPc macrocycle

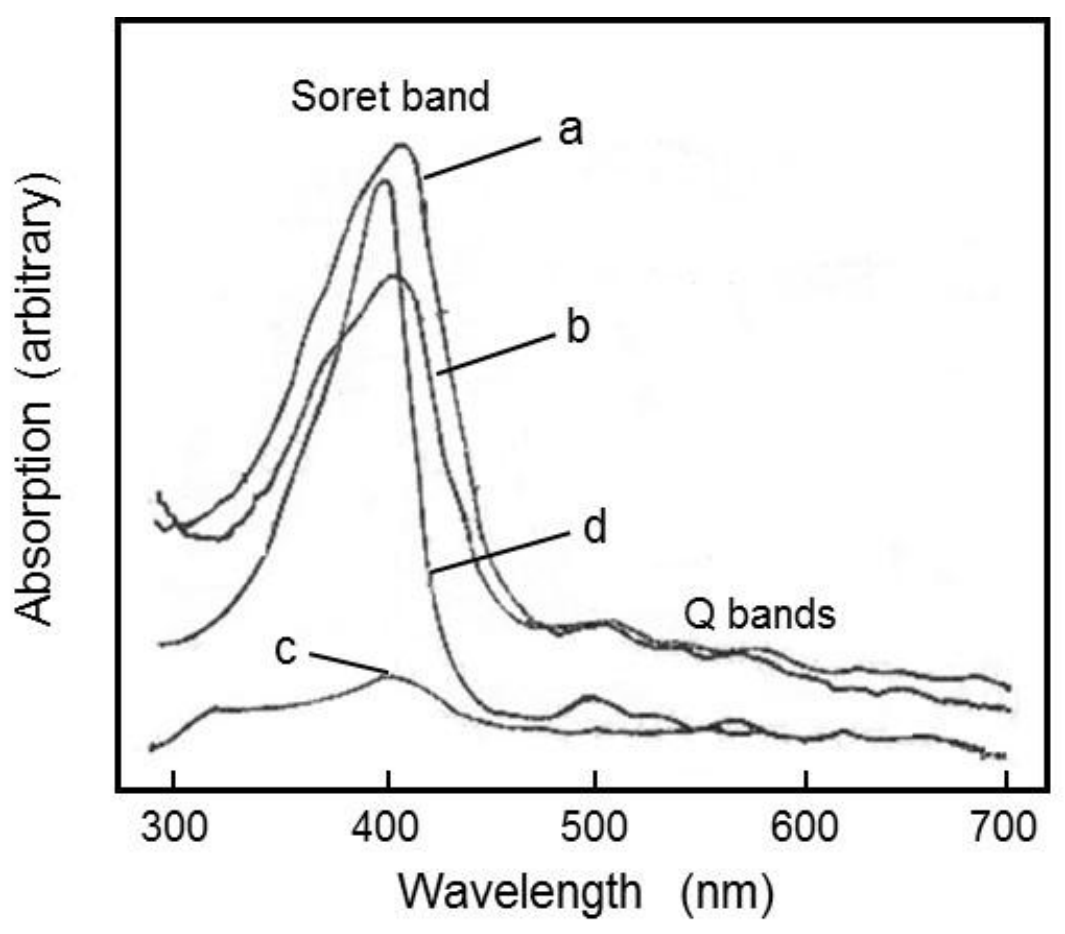

Fig. 3 UV-vis absorption spectra of Porphyrin C in multilayers and solution. a. 17 layers; b. 11 layers; c. 5 layers; d. solution.

\section{Spectroscopic Study}

One of the striking characteristics of ordered organic molecular films is the orientation of the layer molecule, which determines the functional feature of the films and continues to attract research interests. The plane dichroism of Pc and Por derivative films have further been characterized by us using UV-Vis, IR and fluorescence spectra $[5,9]$. In the case of UV-vis absorption of Phthalocyanine (Pc) and Porphyrin (Por) derivative films, while the number of the layers is below three, the absorption is very weak comparing with that of solution. Representative absorption spectra of 
Porphyrin $\mathrm{C}$ are shown in Fig. 3. The absorption spectra of monolayers and multilayers of porphyrins exhibit a distinct red shift of the Soret band relative to its value in solution samples. There is also a red shift of a smaller magnitude in the $\mathrm{Q}$ bands. The Soret bands exhibit significant broadening compared with those in solution. These shifts and broadenings are typical of aggregates of organic molecules for both porphyrins and phthalocyanines .

\section{Acknowledgements}

Authors would like to thank Prof. W. Q. Chen (Changchun Institute of Applied Chemistry) for his sample support. We acknowledge partial financial supports from Jointly Funded Project of Chinese Civil Aviation Authority and National Natural Science Foundation of China (No. 61179055, No. 51402258), the Science and Technology Planning Project of Guangdong Province (No. 2014A010105056).

\section{References}

[1] K. P. Gentry, G. Thomas: (2009) Phys. Rev. B , 80:174118

[2] A. Capobianchi, A. M. Paoletti, G. Rossi, G. Zanotti, G. Pennesi: ( 2009) Sensors and Actuators B: Chemical 142 (1): 159.

[3] Lu-Lin Li and Eric Wei-Guang Diau, Porphyrin-sensitized solar cells, Chem. Soc. Rev., 2013,42, 291-304

[4] A. W. Snow and N. L. Jarvis: J. Am. Chem. Soc., 1984, 106, 4706

[5] H-N Cui; W. Y.Wang; E. L.Zhou: Thin Solid Films, 1992, 214, 238 242

[6] Y. Zang; W. Q. Chen and Q. Shen: Chem. J.of Chinese Universities, 1993, 11, 1483 and 1995, 11, 547

[7] Y.H. Yang and W. Y. Wang,Spectrosc. Spectral Anal. (China), 5(2)(1985)16-21.

[8] Fujiki, M.; Tabei, H.; Kurihara, T. Langmuir, 1988, 4, 1123-1128

[9] Han Ming Ding, Li Ying Wang, Ying Zhang, Shi Quan Xi,Chinese Chemical Letters, 1997, 8 (5), 453-454 